\title{
Deferoxamine-Induced Migration and Odontoblast Differentiation via ROS- Dependent Autophagy in Dental Pulp Stem Cells
}

\author{
Xuan Wang Tian Tian Wu Long Jiang Du Rong Ya Qin Zhu \\ Department of General Dentistry, Ninth People's Hospital, Shanghai Jiao Tong University, School of \\ Medicine; Shanghai Key Laboratory of Stomatology \& Shanghai Research Institute of Stomatology, \\ National Clinical Research Center of Stomatology, Shanghai, PR China
}

\section{Key Words}

Regeneration $\bullet$ Cell biology $•$ Cell differentiation $•$ Pulp biology $•$ Endodontics $•$ Odontogenesis

\begin{abstract}
Background/Aims: As a vital degradation and recycling system, autophagy plays an essential role in regulating the differentiation of stem cells. We previously showed that iron chelator deferoxamine (DFO) could promote the repair ability of dental pulp stem cells (DPSCs). Here, we investigated the effect of DFO in autophagy and the role of autophagy in regulating the migration and odontoblast differentiation of DPSCs. Methods: Transmission electron microscopy, immunofluorescence staining and western blotting were performed to evaluate the autophagic activity of DPSCs. Transmigration assay, alkaline phosphatase staining/activity, alizarin red S staining and quantitative PCR were performed to examine the migration and odontoblast differentiation of DPSCs. Reactive oxygen species (ROS) levels and the effects of ROS scavenger in autophagy induction were also detected. Autophagy inhibitors (3MA and bafilomycin A1) and lentiviral vectors carrying ATG5 shRNA sequences were used for autophagy inhibition. Results: Early exposure to DFO promoted the mineralization of DPSCs and increased autophagic activity. Autophagy inhibition suppressed DFO-induced DPSC migration and odontoblast differentiation. Furthermore, DFO treatment could induce autophagy partly through hypoxia-inducible factor $1 \alpha / B$ cell lymphoma $2 /$ adenovirus $E 1 B$ 19K-interacting protein 3 (HIF-1 $\alpha / B N I P 3)$ pathway in a ROS-dependent manner. Conclusion: DFO increased DPSC migration and differentiation, which might be modulated through ROSinduced autophagy.

\section{Introduction}

Stem cells play an indispensable role in tissue engineering for the reconstruction of natural tissues [1]. Due to the easily accessible source of dental tissues, dental-derived stem

Ya Qin Zhu

KARGER
Department of General Dentistry, Ninth People's Hospital, Shanghai Jiao Tong

University, and National Clinical Research Center of Stomatology, No. 639, Zhi Zao Ju

Road, Shanghai, (China)

Tel. +86-21-23271699-5542, Fax +86-21-63135412, E-Mail zyq1590@163.com 


\section{Cellular Physiology Cell Physiol Biochem 2017;43:2535-2547

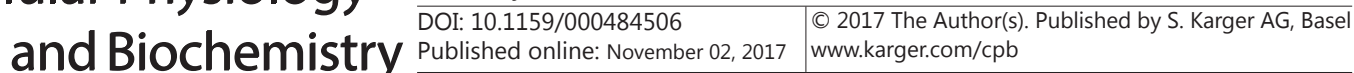 \\ Wang et al.: Deferoxamine-Induced Autophagy in the Repair Ability of Dental Pulp Stem}

Cells

cells are considered suitable candidates for tissue engineering applications [1, 2]. Dental pulp stem cells (DPSCs), isolated from human dental pulp tissue, possess multilineage differentiation potential, including osteogenic, dentinogenic, adipogenic, chondrogenic, myogenic, and neurogenic differentiation [3]. Reflecting its special anatomical structure, dental pulp is often exposed to hypoxic conditions [4]. In previous studies, we showed that hypoxia could up-regulate the expression of mineralized markers and promote the formation of calcified nodules in DPSCs [5]. Following this study, we discovered a positive effect of deferoxamine (DFO) in the repair ability of DPSCs [6]. DFO, extensively utilized as a hypoxia mimetic agent, is an iron chelator clinically used in treating iron overload, which could sequester labile iron (Fe) and attenuate the degradation of HIF-1a [7]. However, the molecular mechanism involved in the effect of DFO in the repair ability of DPSCs remains largely unknown.

Autophagy, regulated by a series of autophagy-related gene (ATG) products, is an evolutionarily conserved pathway involved in the degradation and recycling of cellular proteins and organelles [8]. Autophagic process requires the formation of a doublemembrane autophagosome, and ultimately the autophagosome fuses with the lysosome to form an autophagolysosome to degrade the cargo proteins or organelles [8]. As a recycling system, autophagy plays a vital role in the renovation of cells and tissues [9]. Several studies have implicated the involvement of autophagy in different physiological process of DPSCs, such as tooth development [10], cellular homeostasis [11], apoptosis [12] and senescence [13]. Recently, there is emerging evidence implicating autophagy as an essential role for bone remodeling and osteoblastic differentiation [14]. Considering that bone and dentin share some components and properties, it is attractive to explore the potential effects of autophagy in the differentiation and mineralization of DPSCs.

Recent studies have demonstrated that DFO could induce autophagy via diverse pathways [15]. Chung et al. discovered that DFO treatment could promote the generation of Reactive Oxygen Species (ROS) through sequestering intracellular iron in normal cells [16]. It is well established that ROS is one prominent inducer for autophagy. Our previous study demonstrated that DFO could induce the up-regulation of hypoxia-inducible factor $1 \alpha$ (HIF-1 $\alpha$ ) in DPSCs [6].Moreover, a series of studies have indicated that mitochondrial ROS is involved in the activation of HIF-1 $\alpha$, and HIF- $1 \alpha$ plays a crucial role in HIF-1-dependent autophagy $[17,18]$. Therefore, we hypothesized that DFO might promote the repair ability of DPSCs by inducing autophagy in part through ROS/HIF-1 $\alpha$ pathway. In the present study, we explored the effects of DFO in the autophagic activity of DPSCs and the role of autophagy in DFO-induced migration, differentiation and mineralization, and investigated the autophagic mechanisms in DFO-treated DPSCs.

\section{Materials and Methods}

Cell Culture, DFO and Iron Treatment, Odontoblastic Induction, and Autophagy Inhibitior Treatment

Nine intact third molars were collected from healthy patients between 18 and 22 years old. Approval for the whole research was obtained from the Ethics Committee of our hospital and the patients provided informed consent. DPSCs were isolated from dental pulp using the outgrowth method, and the cells were subsequently cultured in alpha-minimum essential medium ( $\alpha$-MEM; HyClone, Logan, UT, USA) containing $10 \%$ FBS according to a previous study $[19,20]$. For DFO and iron treatment, DPSCs were incubated in culture medium containing different concentrations of DFO or DFO with ferric citrate (FC) at the indicated time. For odontoblastic induction, the cells were cultured in mineralized-induced $\alpha$-MEM medium containing $10 \%$ FBS, $50 \mu \mathrm{g} / \mathrm{ml}$ ascorbic acid, $10 \mathrm{mM}$ sodium $\beta$-glycerophosphate and $10 \mathrm{nM}$ dexamethasone (Sigma-Aldrich Inc., St. Louis, MO, USA). Autophagy inhibitiors was applied in the study as follows: $5 \mathrm{mM}$ of 3-mathyladenine (3-MA) (Sigma-Aldrich Inc.) and $10 \mathrm{nM}$ of bafilomycin A1 (BA1) (Santa Cruz Biotechnology, Inc.).

Lentivirus Transfection-driven ATG5 Knockdown

ATG5 expression was knocked down using short hairpin RNA (shRNA). The ATG5 silencing sequence (ATG5 sh RNA 5'-GCAGTGGCTGAGTGAACAT-3') was subcloned into the pLKD-CMV-G\&PR-U6-shRNA 


\section{Cellular Physiology Cell Physiol Biochem 2017;43:2535-2547 \\ \begin{tabular}{l|l|l} 
and Biochemistry $\begin{array}{l}\text { DOI: 10.1159/000484506 } \\
\text { Published online: November 02, } 2017\end{array}$ & $\begin{array}{l}\text { (c) } 2017 \text { The Author(s). Published by S. Karger AG, Basel } \\
\text { www.karger.com/cpb }\end{array}$
\end{tabular} \\ Wang et al.: Deferoxamine-Induced Autophagy in the Repair Ability of Dental Pulp Stem}

Cells

lentiviral vector (Oobio, Shanghai, China). The empty vector was used as a negative control. Each construct, together with helper vectors was transfected into the $293 \mathrm{~T}$ cells. After $72 \mathrm{~h}$, the viruses were collected and transfected into DPSCs. The representative imaging of DPSCs transfected with ATG5shRNA was shown (for all online suppl. material, see www.karger.com/doi/10.1159/000484506) in Suppl. Fig. 1.

\section{Cell Viability Assay}

Cell viability assays were performed using methylthiazol tetrazolium (MTT; Amresco, Solon, $\mathrm{OH}$ ) assay. DPSCs at a concentration of $3 \times 10^{3}$ cells were plated onto 96-well plates. After overnight incubation, the DPSCs were treated with conditioned medium for the indicated times. Subsequently, $20 \mu \mathrm{l}$ of MTT solution was added to each well. After incubation for $4 \mathrm{~h}$ at $37^{\circ} \mathrm{C}$, the medium containing MTT was discarded, and $150 \mu \mathrm{l}$ of dimethyl sulfoxide (Sigma-Aldrich Inc.) was added to each well. The optical density (OD) of each well was measured using an enzyme linked immune detector at a wavelength of $490 \mathrm{~nm}$. All experiments were performed in triplicate.

\section{Transmigration Assay}

Transwell inserts (8-mm pore, Corning) were used for transmigration assay. DFO was added in the lower chamber, while $5 \times 10^{3}$ cells which had been pre-incubated with autophagy inhibitors or lentiviral vectors were added to the upper chamber. After $24 \mathrm{~h}$, DPSCs were fixed with $4 \%$ paraformaldehyde and then stained with crystal violet (Sigma-Aldrich Inc., St. Louis, MO, USA) for visualization of migrated cells. Cell number counting was implemented in three random high-power fields to assess the average number of migrating cells.

\section{Alkaline Phosphatase (ALP) Staining/Activity and Alizarin Red S (ARS) Staining}

DPSCs were pretreated with culture medium containing DFO, DFO+3-MA and DFO+BA1 or transfected with lentivirus for ATG5 knockdown. Subsequently, the conditioned medium was removed, and mineralized medium were added to the well. The ALP staining/activity assay was performed using an ALP staining kit (86R-1KT; Sigma-Aldrich, Inc.) on days 3 and 7 according to the manufacturer's protocol. For the quantitative ALP assay, the cells were detected after measuring the optical density (OD) values at $405 \mathrm{~nm}$ after incubation with p-nitrophenyl phosphate (Sigma-Aldrich, Inc.). ARS staining was performed on day 21. The cells were fixed with $70 \%$ ethanol and treated with $2 \%$ alizarin red (pH 4.2, Sigma-Aldrich, Inc.). All staining images were photographed using a microscope and subsequently analyzed using an HP Officejet Pro L7580 scanner. ARS-stained cells were subsequently destained in 10\% acetylpyridinium chloride (Sigma-Aldrich, Inc.) and measured at $590 \mathrm{~mm}$ using a microplate reader. For both ALP and ARS quantitative assays, the total protein content of the cells was measured, and the results were expressed as OD values per milligram of total protein. All treatments were performed in the initial days, except for special instruction. To investigate the effect of DFO at different stage of differentiation, DFO treatment was performed on days 1-2, 11-12 or 20-21 (see online suppl. material, Suppl. Fig. 2).

\section{Transmission Electron Microscope (TEM)}

DPSCs were fixed with $2 \%$ glutaraldehyde in $0.1 \mathrm{M}$ cacodylate buffer for $1 \mathrm{~h}$ at $4{ }^{\circ} \mathrm{C}$ and post-fixed in $1 \%$ osmium tetroxide in the same buffer for $1 \mathrm{~h}$ at room temperature. After dehydration through a graded series of ethanol, the samples were embedded in Epon. Ultrathin sections were stained with $2 \%$ uranyl acetate and lead citrate. The electron microscopy was conducted using a JEM-1200EX Transmission Electron Microscope (JEOL, Tokyo, Japan).

\section{Immunofluorescence Staining}

DPSCs were seeded onto coverslips in a 6-well plate. After overnight incubation, the DPSCs were treated with conditioned medium for the indicated times. After removing the medium, the cells were washed with PBS and fixed with $4 \%$ paraformaldehyde for 20 min. Following permeabilization with $0.5 \%$ Triton X-100 for $15 \mathrm{~min}$, the cells were incubated with goat serum for $1 \mathrm{~h}$. For LC3 localization and detection, the cells were incubated with rabbit anti-LC3B antibody (1:500, Sigma-Aldrich Inc., St. Louis, MO, USA) overnight. Subsequently, the DPSCs were treated with FITC-conjugated goat anti-rabbit IgG (1:50, Beijing Cwbio Co., Beijing, China) for $1 \mathrm{~h}$ and counterstained with DAPI for $5 \mathrm{~min}$. The photographs were captured using a Nikon fluorescence microscope. 


\section{Cellular Physiology Cell Physiol Biochem 2017;43:2535-2547 and Biochemistry Published online: November 02, $2017 \begin{aligned} & \text { DOI: 10.1159/000484506 } 2017 \text { The Author(s). Published by S. Karger AG, Basel } \\ & \text { www.karger.com/cpb }\end{aligned}$ \\ Wang et al.: Deferoxamine-Induced Autophagy in the Repair Ability of Dental Pulp Stem \\ Cells}

\section{Western Blot Analysis}

The cell samples were harvested and washed with PBS on ice and subsequently lysed in lysis buffer (RAPA:PMSF=100:1). The concentrations of proteins were detected using a BCA protein assay kit (BioRad). The proteins were separated on SDS-polyacrylamide gels and transferred to PVDF membranes. The membranes were incubated with primary antibodies overnight against LC3b (Sigma-Aldrich Inc.) at a dilution of 1:750, SQSTM1/p62 (Sigma-Aldrich Inc.) , Beclin1 (Sigma-Aldrich, Inc.) at a dilution of 1:1000, HIF-1a (Sigma-Aldrich, Inc.) at a dilution of 1:1000, and B cell lymphoma 2/adenovirus E1B 19K-interacting protein 3 (BNIP3) (Santa Cruz Biotechnology, Inc.) at a dilution of 1:1000. Subsequently, the membranes were incubated with secondary antibodies for $60 \mathrm{~min}$, and the blotted bands were visualized after ECL exposure to X-ray. The band density was analyzed using Scion Image-Release Beta 4.02 software.

\section{Real-time PCR assay}

Total RNA was isolated from DPSCs using Trizol (Invitrogen, Carlsbad, CA), and cDNA was created using the RT Reagent Kit (Takara, Shiga, Japan) as previously described [6]. Quantitative PCR was performed with SYBR Green Mix (Takara, Shiga, Japan) on a Roche Light Cycler 480 (Roche Diagnostics GmbH, Mannheim, Germany) in a total reaction volume of $20 \mu$ l. The expressions of several gene markers, including dentin sialophosphoprotein (DSPP), dentin matrix protein-1 (DMP-1), bone sialoprotein (BSP), collagen type I (COL-I) and runt-related transcription factor 2 (Runx2), were detected, and the gene specific primers are listed (see online suppl. material) in Suppl. Table 1. The mRNA expression levels of these genes were quantified using the comparative threshold cycle (2_ $\Delta \Delta \mathrm{CT})$ method.

\section{Assessment of ROS generation}

Intracellular ROS generation of DPSCs was measured using a 5-(and-6)-chloromethyl-2', 7'-dichlorodihydro-fluorescein diacetate, acetylester fluorescent probe (CM-H2DCFDA, Sigma-Aldrich, Inc.). The cells were treated with DFO for $24 \mathrm{~h}$ and subsequently incubated with $10 \mu \mathrm{M} \mathrm{CM-H2DCFDA}$ for 20 min at $37^{\circ} \mathrm{C}$ in the dark. ROS formation was analyzed using a FACSCalibur flow cytometer (Becton Dickinson, San Jose, CA, USA).

\section{Statistical Analysis}

The outcome data were expressed as the means \pm standard deviation of at least three independent experiments. Statistical analysis was performed using one-way analysis of variance, followed by Bonferroni's post hoc test for multiple comparisons. Values of $\mathrm{p}<0.05$ were considered statistically significant.

\section{Results}

\section{Early Exposure to DFO Promoted the Mineralization of Dental Pulp Stem Cells}

To determine the duration of DFO treatment, we detected the cell viability of DPSCs treated with various concentrations $(0-50 \mu \mathrm{M})$ of DFO on day 1 , day 2 and day 3 . As shown in Fig. 1D, the viability of DPSCs was not affected by different concentrations of DFO until the 3rd day. On day 3, the viability of DPSCs was significantly decreased compared with the control group. Thus 48 hours of DFO treatment was selected for further ALP and ARS staining. Consistent with our previous study, DFO-treated group showed increased ALP staining/ activity (at day 3 and day 7, Fig. 1A). DFO treatment at the initial stage of differentiation (DFO-I group) markedly increased ARS staining as shown in Fig. 2B, while DFO treatment performed at the medial (DFO-M group) and late stage (DFO-L group) of differentiation did not promote the mineralization of DPSCs (Fig. 1B), suggesting that DFO treatment is more effective in promoting the mineralization of DPSCs at the earlier stages of differentiation. In addition, a longer DFO culture period (3-7 days) did not increase the amounts of mineralized nodules (data not shown). Representative enlarged calcified nodule induced by DFO treatment in DPSCs was shown in Fig. 1C. 


\section{Cellular Physiology and Biochemistry \begin{tabular}{l|l} 
DOI: 10.1159/000484506 & (c)17 The Author(s). Published by S. Karger AG, Basel \\
www.karger.com/cpb
\end{tabular}

Fig. 1. Early exposure to deferoxamine (DFO) promoted the mineralization of dental pulp stem cells (DPSCs). (A) DPSCs were treated with or without DFO $(10 \mu \mathrm{M})$ for 2 days, and subsequently cultured in mineralization induction medium. ALP staining and ALP activity in the control and DFO group on days 3 and 7. (B) DPSCs were treated with DFO (10 $\mu \mathrm{M}$ ) at the initial (day 1-2, DFO-I), medial (day 11-12, DFO-M) or late (day 2021, DFO-L) stages of mineralization. Alizarin red S staining and quantitative mineralization assay of the four groups (control, DFO-I, DFO-M and DFO-L) on day 21. (C) Representative enlarged calcified

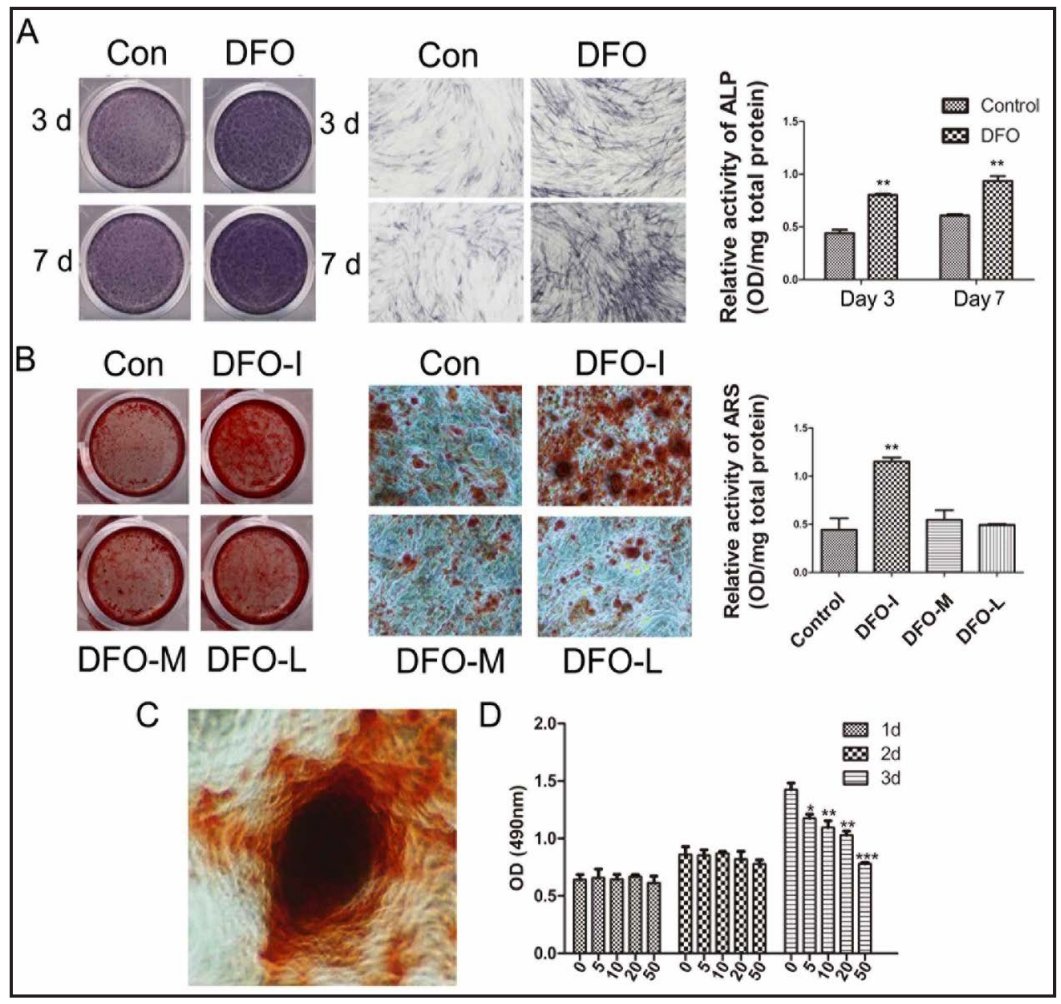
nodule induced by DFO treatment in DPSCs. (D) DPSCs were treated with different concentrations $(0,5,10$, 20 and $50 \mu \mathrm{M}$ ) of DFO for 1,2 and 3 days. Cell viability was measured using an MTT assay. ${ }^{*} \mathrm{p}<0.05$, versus control group; ${ }^{* *} \mathrm{p}<0.01$, versus control group; ${ }^{* * *} \mathrm{p}<0.001$, versus control group.

\section{DFO-induced Activation of Autophagy in Dental Pulp Stem Cells}

To explore whether DFO could induce autophagy in DPSCs, we detected the formation of autophagic elements and autophagic flux. For the detection of autophagic elements, we used morphological analyses to examine autophagosome formation. LC3B is a common marker for autophagosome formation [21], and immunostaining with LC3B was performed in the present study. As shown in Fig. 2A, DFO treatment significantly increased the number of LC3B puncta, suggesting the formation of autophagosomes after $24 \mathrm{~h}$ and $48 \mathrm{~h}$. To further confirm the effect of DFO on autophagic activity, a TEM analysis was performed at an ultrastructural level. The presence of autophagosome or initial autophagic vacuoles (AVi) and degradative autophagic vacuoles (AVd) was observed in the DFO-treated group (Fig. 2B). Notably, autophagosome formation differs from increased autophagic activity. A block in the late stages of autophagy could also result in autophagosome formation. In the present study, we performed western blotting to detect the autophagy-related protein expression of LC3B, SQSTM1/p62 (p62) and Beclin 1 during DFO treatment. LC3B and Beclin 1 are both common markers for monitoring autophagy, and p62 is an autophagy cargo receptor for the delivery of aggregated proteins for degradation. The decreased expression of p62 is often associated with increasing autophagic flux [22]. The results of western blotting showed that DFO treatment obviously increased the protein expression of LC3B-II and Beclin 1 and gradually decreased the protein level of p62 (Fig. 2C). After blocking autophagy in the late stage by BA1, DFO treatment further upregulated LC3B-II expression in DPSCs compared with cells treated with DFO alone (Fig. 2F). To further demonstrate that iron chelator (DFO) could induce autophagy in DPSCs, autophagic level was detected in addition of FC (Fe iron). As shown in Fig. 2D, DFO-induced autophagy was blocked by addition of FC, suggesting that DFO could induce autophagy via sequestering intracellular iron. Interestingly, excessive iron 


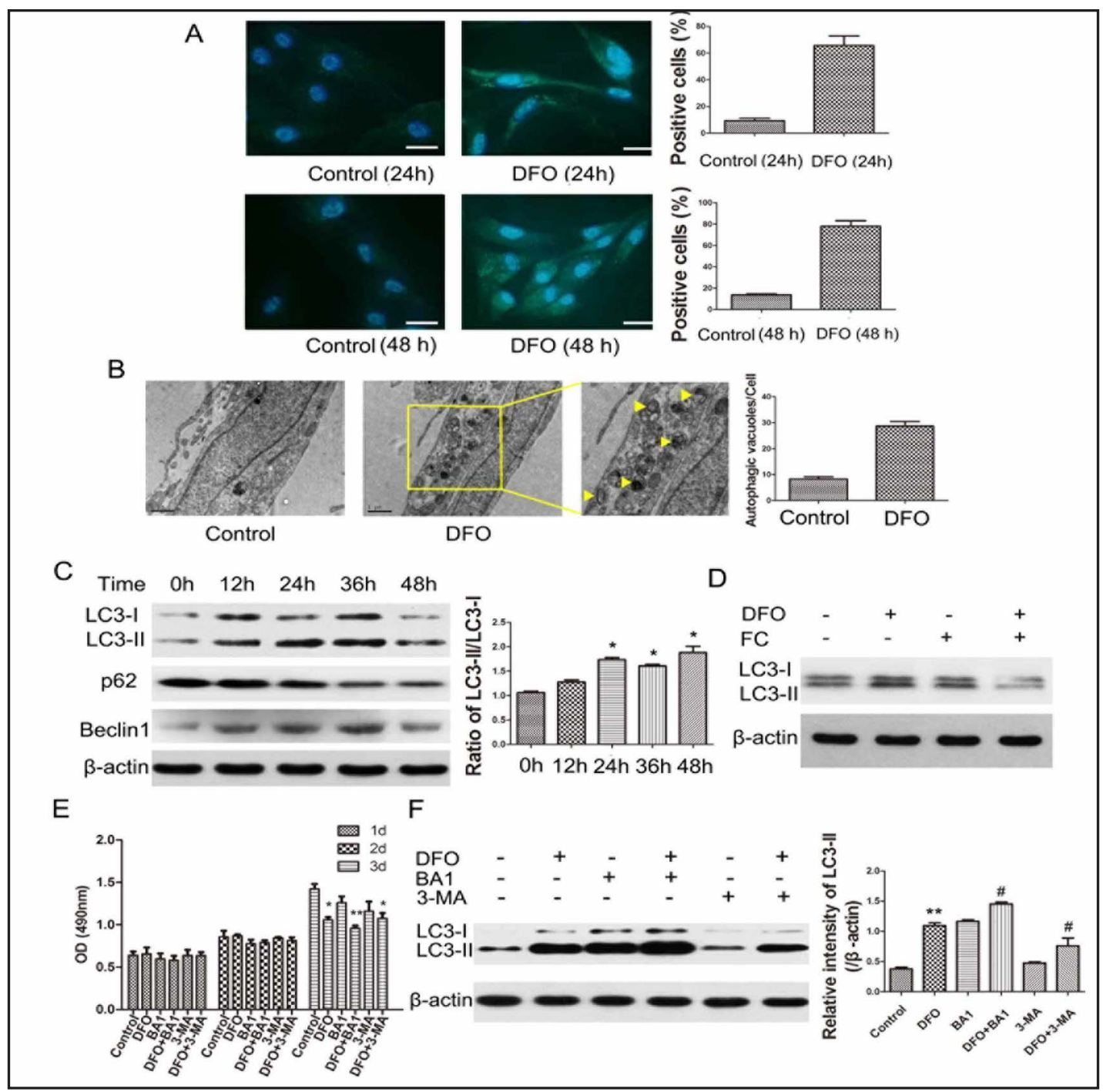

Fig. 2. Deferoxamine-induced activation of autophagy in dental pulp stem cells (DPSCs). (A) DPSCs were treated with deferoxamine (DFO, $10 \mu \mathrm{M}$ ) for the indicated times. Merged pictures of immunofluorescence for LC3B and DAPI staining in DPSCs with or without DFO treatment. Increased numbers of LC3B puncta were detected in DFO-treated DPSCs compared with the control (scale bar, $10 \mu \mathrm{m}$ ). (B) Representative transmission electron microscope images of DPSCs treated with or without DFO. Yellow arrows indicate typical autophagic vacuoles. (C) The protein expression of LC3B, SQSTM1/p62 and Beclin 1 was detected using western blotting. The ratio of LC3-II/LC3-I was shown. (D) Addition of Fe iron attenuated the protein expression of LC3B-II induced by DFO. (E) The graph shows the ratio of LC3-II/LC3-I. (E) DPSCs were treated with cultured medium, deferoxamine (DF0, $10 \mu \mathrm{M}$ ), bafilomycin A1 (BA1), DF0+BA1, 3-mathyladenine (3-MA) and 3-MA+DFO for $24 \mathrm{~h}$. Cell viability in all groups from $24 \mathrm{~h}$ to 3 days. (F) Western blotting for LC3B showed autophagy suppression by autophagy inhibitors. ${ }^{*} \mathrm{p}<0.05$, versus control group; ${ }^{* *} \mathrm{p}<0.01$, versus control group; ${ }^{\mathrm{p}}<0.05$, versus DFO group

could also up-regulate the expression of LC3B (Fig. 2D), indicating that autophagy is closely associated with iron metabolism.

Inhibition of Autophagy Suppressed DFO-induced Dental Pulp Stem Cell Migration

Autophagy inhibition was achieved by using autophagy inhibitors and ATG5 knockdown. The cell viability of DFO-treated DPSCs was not affected in the presence of autophagy 


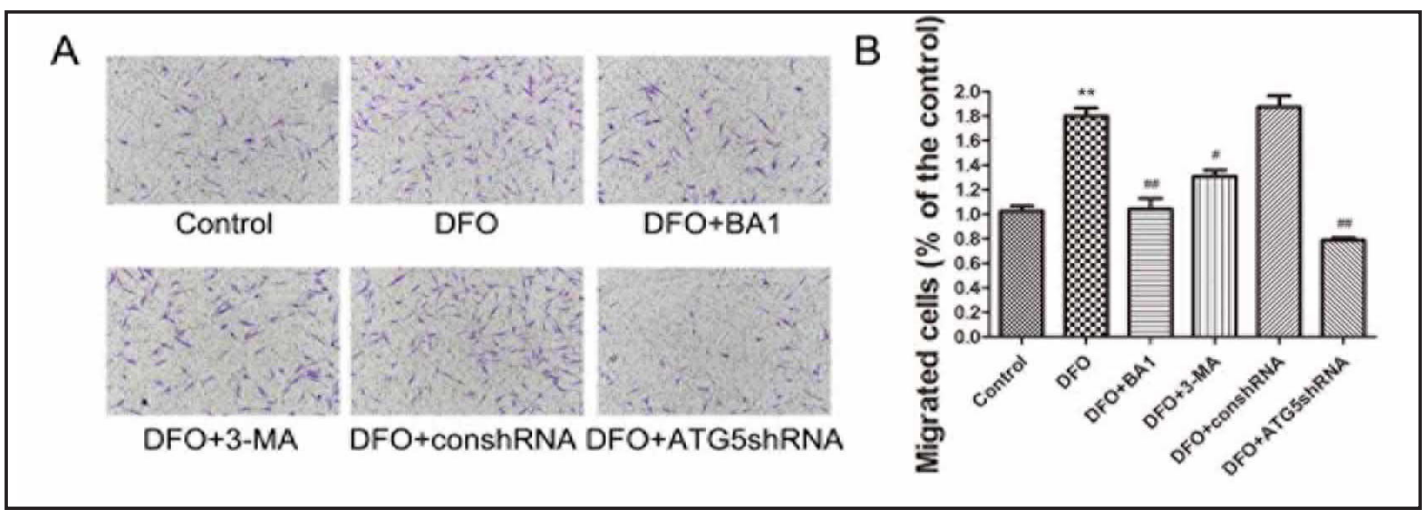

Fig. 3. Inhibition of autophagy suppressed deferoxamine-induced dental pulp stem cell (DPSC) migration. DPSCs were treated with cultured medium, deferoxamine (DFO, $10 \mu \mathrm{M}$ ), DFO+bafilomycin A1 (BA1), DFO+3mathyladenine (3-MA) for $24 \mathrm{~h}$, and the effect of DFO in the migration of DPSCs was also examined in DPSCs transefected with ATG5 shRNA. (A) Representative images of the migrated cells in different groups. (B) The graph shows the number of migrated cells in different groups. ${ }^{* *} \mathrm{p}<0.01$, versus control group; ${ }^{*} \mathrm{p}<0.05$, versus DFO group, ${ }^{\# \#} \mathrm{p}<0.01$, versus DFO group.

inhibitors (Fig. 2E), and western blotting for LC3B demonstrated the inhibition of autophagy in the presence of early stage autophagy inhibitor (3-MA) and late stage autophagy inhibitor (BA1) (Fig. 2F). ATG5 is a essential gene for autophagy induction, and knockdown of ATG5 has been widely used for autophagy inhibition [22]. ATG5 knockdown was confirmed by real-time quantitative polymerase chain reaction (PCR) and Western blotting analysis (see online suppl. material, Suppl. Fig. 3).

Transmigration assay was performed to detect the migration of DPSCs. As shown in Fig. 3 , the migrated DPSCs was significantly increased after incubation with DFO. Autophagy inhibition by autophagy inhibitors and ATG5 shRNA both remarkably suppressed DFOinduced migration as presented in Fig. 3. These results indicated the involvement of autophagy in DFO-induced migration.

\section{Inhibition of Autophagy Suppressed DFO-induced Dental Pulp Stem Cell Differentiation} and Mineralization

To investigate the role of autophagy in DFO-induced mineralization, an ALP staining/ activity assay and ARS staining were performed. ALP activity of DFO-treated DPSCs was inhibited in the presence of autophagy inhibitors on day 7 (Fig. 4A). After induction for 3 weeks, the amount of minerals detected remarkably decreased with the addition of autophagy inhibitors compared with DFO treatment alone (Fig. 4B). These results suggested that autophagy is essential for DFO-induced mineralization. To investigate the influence of autophagy on DFO-induced differentiation, the mRNA expression of odontogenesis/ osteogenesis-related molecules in DPSCs was detected. Similarly, real-time PCR assay revealed that the inhibition of autophagy using 3-MA or BA1 suppressed the expression of several gene markers, including DSPP, DMP-1, BSP, COL-I and Runx2 (Fig. 4C). These data suggested an essential role for autophagy in DFO-induced differentiation and mineralization.

ATG5 knockdown Suppressed DFO-induced Dental Pulp Stem Cell Differentiation and Mineralization

To further confirm the role of autophagy in DFO-induced mineralization, we blocked autophagic process by ATG5 knockdown. ALP activity and ARS staining were measured in DPSCs transfected with ATG5 shRNA. As shown in Fig. 5A, ATG5 knockdown blocked DFOinduced ALP activity compared with the control and control shRNA groups. Alizarin red S staining indicated that the amount of mineralized nodules was observed less in the shRNA groups (Fig. 5B). PCR assay for mRNA expression of odontogenesis/osteogenesis-related 


\section{Cellular Physiology

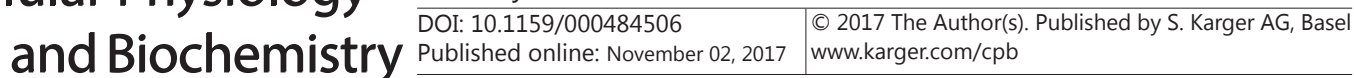

Fig. 4. Inhibition of autophagy suppressed deferoxamine-induced dental pulp stem cell (DPSC) differentiation and mineralization.(A) DPSCs were treated with cultured medium, deferoxamine (DFO, 10 $\mu \mathrm{M})$, DFO+BA1 or $\mathrm{DFO}+3-$ $\mathrm{MA}$ at initial stage during the mineralized-induction. ALP staining and activity of four groups (control, DFO, DFO+BA1 and DFO+3-MA) on day 7. (B) Alizarin red $\mathrm{S}$ staining and quantitative mineralization assay of the four groups (control, DFO, DFO+BA1 and DFO+3-MA) on day 21. (C) The odontogenesis/osteogenesis-related gene (DSPP, DMP-1, BSP, COL-1, RUNX2) expression in the four groups (control, DFO, DFO+BA1 and DFO+3MA) on day $7 .{ }^{*} p<0.05$, versus control group; ${ }^{* *} \mathrm{p}<0.01$, versus control group; ${ }^{\#} \mathrm{p}<0.05$, versus DFO group; ${ }^{\# \#} \mathrm{p}<0.01$, versus DFO group.

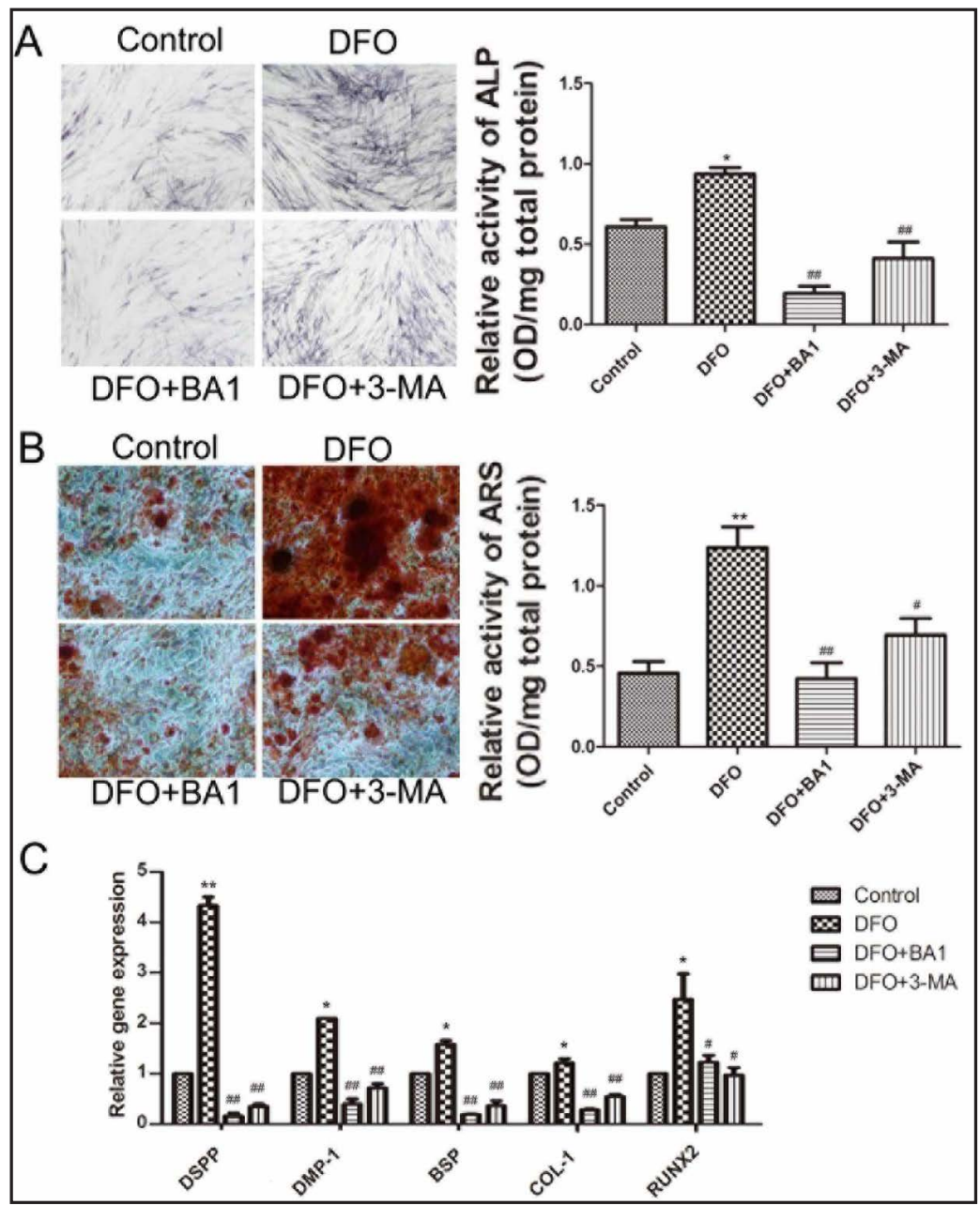

molecules showed that autophagy inhibition by ATG5 knockdown blocked DFO-induced differentiation (Fig. 5C). All these data indicated that autophagy inhibition by inhibitors or silencing crucial autophagy proteins blocked the positive effects of DFO in DPSCs, and demonstrated that autophagy plays a vital role in DFO-induced mineralization and differentiation.

\section{DFO Induced Autophagy through Regulating ROS/HIF-1a/BNIP3 pathway in Dental Pulp} Stem Cells

To explore the potential molecular mechanism underlying DFO-induced autophagy, we detected intracellular ROS generation in DPSCs after exposure to DFO. As shown in Fig. 6A, ROS levels were increased after incubation with DFO for $24 \mathrm{~h}$. The ROS scavenger N-acetylL-cysteine (NAC) was used to determine the role of ROS in DFO-induced autophagy. NAC significantly suppressed the generation of ROS and the expression of LC3B-II in the presence of NAC compared with DFO treatment alone, indicating that DFO might induce autophagy through ROS generation (Fig. 6B). Our previous study showed that DFO could increase the expression of HIF-1a [6]. We hypothesized that DFO could mediate the expression of HIF1a through ROS generation. As shown in Fig. 6C, HIF-1a was up-regulated in DFO-treated groups, and NAC suppressed the expression of HIF-1a in DFO-treated groups, suggesting that HIF-1a was regulated by ROS generation. It is well established that HIF-1a is crucial for hypoxia-induced autophagy and could induce autophagy through HIF-1a/BNIP3 pathway. BNIP3, a hypoxia-responsive gene directly regulated by HIF-1a, has been identified as one of the most prominent autophagic genesis [23]. Thus, we evaluated the expression of HIF-

\section{KARGER}




\section{Cellular Physiology and Biochemistry \begin{tabular}{l|l} 
DOI: 10.1159/000484506 2017 The Author(s). Published by S. Karger AG, Basel \\
wuww.karger.com/cpb
\end{tabular}

Fig. 5. Knockdown of ATG5 suppressed deferoxamineinduced dental pulp stem cell (DPSC) differentiation and mineralization.(A) DPSCs were treated with deferoxamine (DFO, $10 \mu \mathrm{M}$ ) for DFO treatment. The effect of DFO was examined in control DPSCs, DPSCs transfected with conshRNA and ATG5shRNA. ALP staining and activity of four groups (control, DFO, DFO+conshRNA and DFO+ATG5shRNA) on day 7. (B) Alizarin red S staining and quantitative mineralization assay of the four groups (control, DFO, DFO+conshRNA and DFO+ATG5shRNA) on day 21. (C) The odontogenesis/osteogenesis-related gene (DSPP, DMP-1, BSP, COL-1, RUNX2) expression in the four groups (control, DFO, DFO+conshRNA and DFO+ATG5shRNA) on day $7 .{ }^{*} \mathrm{p}<0.05$, versus control group; ${ }^{* *} \mathrm{p}<0.01$, versus control group; ${ }^{\#} \mathrm{p}<0.05$, versus DFO group; ${ }^{\# \#} \mathrm{p}<0.01$, versus DFO group.

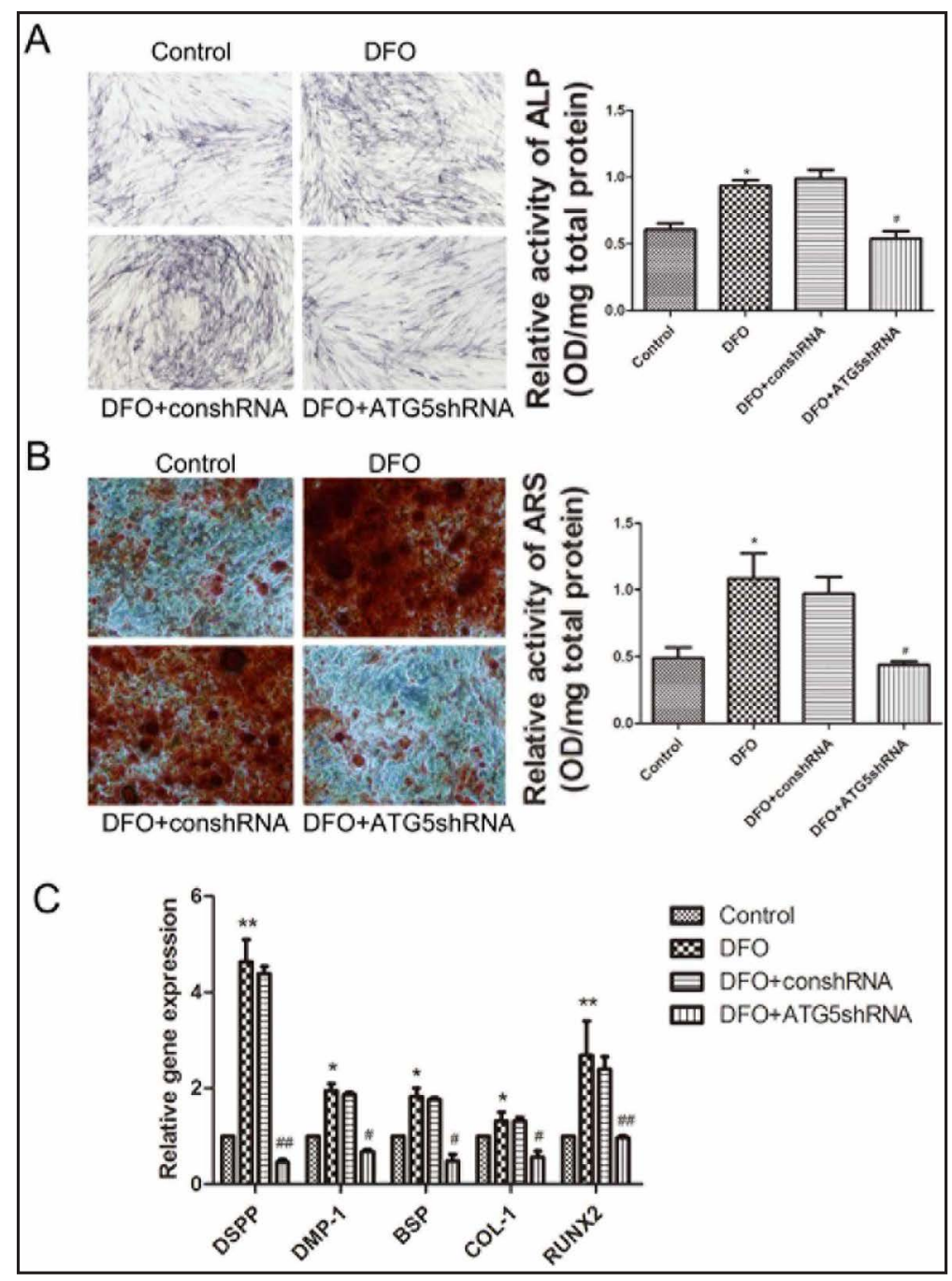

1a/BNIP3 pathway in DFO-treated DPSCs. As shown in Fig. 6D, HIF-1a/BNIP3 pathway was significantly activated after DFO treatment, suggesting that DFO might induce autophagy through HIF-1a/BNIP3 pathway. Moreover, deletion of ROS by NAC also attenuated DFOinduced upregulation of BNIP3, indicating that DFO might induce autophagy through regulating ROS/HIF-1a/BNIP3 pathway.

\section{Discussion}

Dentinal regeneration requires the involvement of several physiological processes in DPSCs, including cell proliferation, migration, angiogenesis, differentiation and mineralization. Effective improvement of the repair ability of DPSCs is of vital importance for its application in tissue engineering. In a previous study, we reported that DFO has could promote the formation of mineralized tissue in DPSCs. In the present study, we demonstrated that DFO could promote the migration, differentiation and mineralization of DPSCs by inducing autophagy, which might be mediated partly through ROS/HIF-1a/BNIP3 pathway.

Iron is an essential element for multiple physiological processes, including oxygen transport, ATP generation, and cellular redox reactions [24]. Previous studies have suggested that iron metabolism is closely associated with bone physiology [25]. Baschant et al. showed that attenuated osteogenic differentiation was observed in the presence of excess iron, whereas elevated osteogenic differentiation was observed in the presence of the 
Fig. 6. DFO Induced Autophagy through Regulating ROS/HIF-1a/ BNIP3 pathway in Dental Pulp Stem Cells(A) DPSCs were treated with cultured medium and different concentrations of deferoxamine (DFO, 10 and $20 \mu \mathrm{M}$ ) and DFO+N-acetyl-L-cysteine (NAC, 5 $\mathrm{mM}$ ) for $24 \mathrm{~h}$ and subsequently loaded with CM-H2DCFDA for 30 min. ROS generation was detected using fluorescence microscopy and flow cytometry. Representative images are presented, and the graph shows the quantitative analysis of ROS generation. (B) For ROS scavenging, DPSCs were preincubated with NAC for $2 \mathrm{~h}$, and subsequently treated with DFO for $24 \mathrm{~h}$. Western blotting for LC3B was performed in the four groups (control, NAC, NAC+DFO and DFO). The graph shows the relative intensity of the proteins. (C) The protein expression of HIF1a/BNIP3 pathway in in the four groups (control, DFO, NAC+DFO and NAC). The graph shows the relative intensity of the proteins. (D) Time-dependent protein expression of HIF-1a/BNIP3 pathway in DFO-treated DPSCs. The graph shows the relative intensity of the proteins. ${ }^{*} \mathrm{p}<0.05$, versus control group; ${ }^{* *} \mathrm{p}<0.01$, versus control group; ${ }^{*}<0.05$, versus DFO group; ${ }^{\# \#} \mathrm{p}<0.01$, versus DFO group.

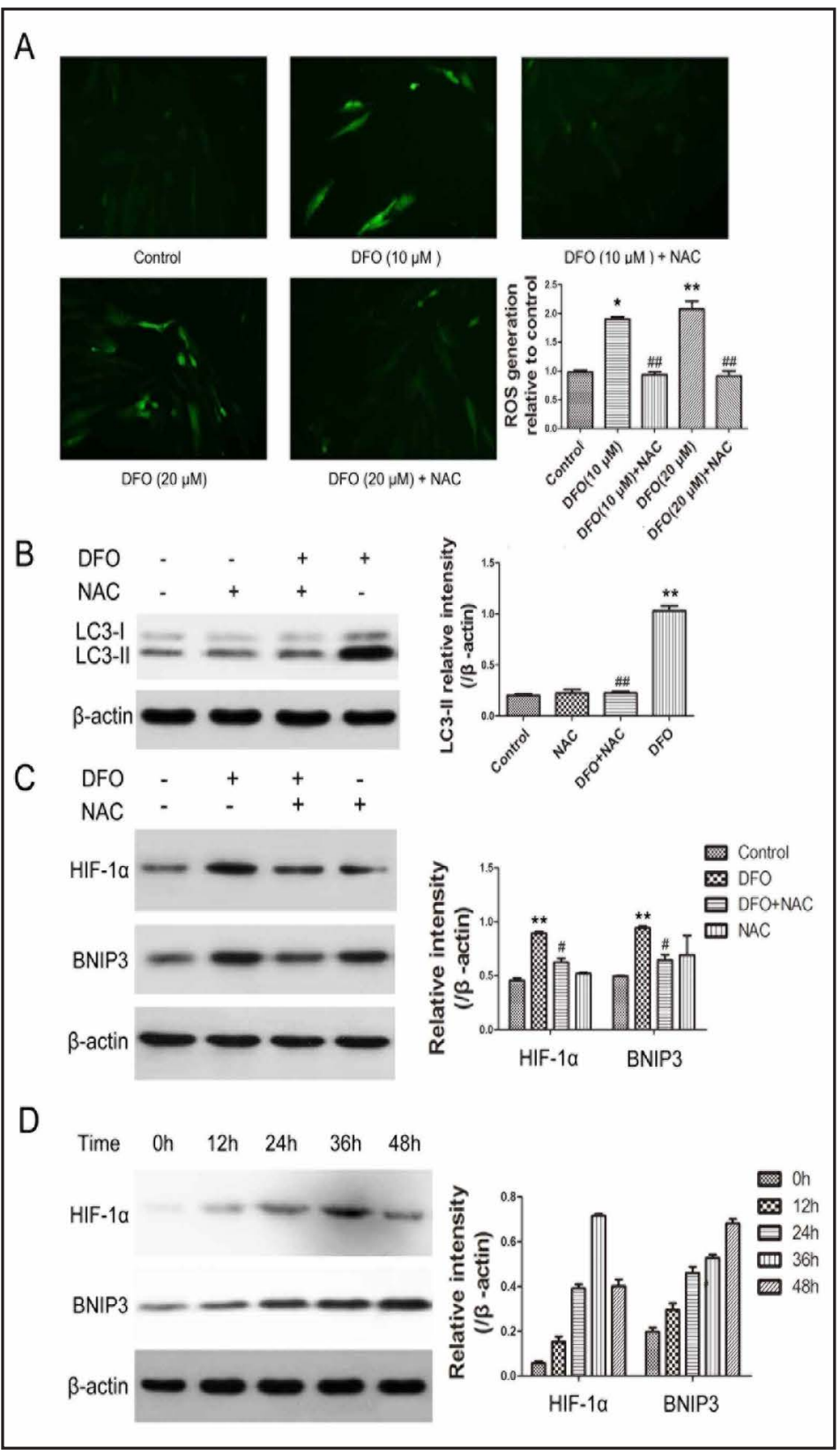

iron chelator (DFO) [25]. Chung et al. discovered that long-term treatment with $200 \mu \mathrm{M}$ DFO could promote the mineralization of human periodontal ligament cells [16]. In the present study, we observed that short-term treatment with a low concentration of iron chelator (10 $\mu \mathrm{M}$ DFO) at the early stages of mineralization could promote the differentiation and mineralization of DPSCs.

The induction of autophagy is closely associated with metabolic stresses, such as reduced energy charge, amino acid depletion, iron depletion, etc. A decrease in the level of intracellular iron by iron chelators could activate the autophagic degradation of ferritin oligomers, which store cytoplasmic iron to maintain the metabolic balance of iron [26]. The effect of DFO in autophagy induction has been confirmed in different cells $[27,28]$. In the present study, we observed increased autophagosome formation and autophagic flux in DFO-treated DPSCs, which is consistent with previous studies.

Autophagy is not only a simple tool for the elimination of cellular materials, but is also an important recycling system for tissue renovation [29-31]. Recently, the role of autophagy

\section{KARGER}




\section{Cellular Physiology Cell Physiol Biochem 2017;43:2535-2547

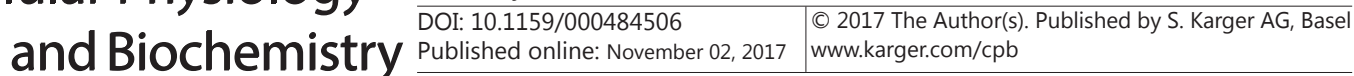 \\ Wang et al.: Deferoxamine-Induced Autophagy in the Repair Ability of Dental Pulp Stem \\ Cells}

in bone physiology and pathophysiology has gradually been unmasked. The effects of autophagy modulators in bone health and osteoblastic differentiation have been widely studied [32], and recent studies have demonstrated that autophagy deficiency compromises osteoblast mineralization in vitro and in vivo $[33,34]$. Because bone and dentin share similar components, it is necessary to investigate the role of autophagy in dentin regeneration and odontoblastic differentiation. Recent studies have discovered that inflammatory cytokine could attenuate the odontoblastic differentiation of dental stem cells [35]. Furthermore, Pei et al. reported that autophagy induced by inflammatory environment is critical in regulating odontoblastic differentiation [36]. More highly reserved ATGs have been identified in regulating the odontoblastic differentiation of stem cells [37]. In the present study, we observed that the regulation of autophagy through both early stage and late stage autophagy inhibitors could alter the effects of DFO-induced migration, differentiation and mineralization in DPSCs. Knockdown of ATG5 resulted in attenuated effects of DFO in the repair ability of DPSCs. These results indicated that autophagy might be a crucial contributor to the repair ability of DPSCs. Further studies are required to understand the pathways between ATGs and mineral markers during pulp responses to DFO.

Previous studies have demonstrated that DFO exposure could induce the generation of reactive oxygen species (ROS) [16]. Elevated ROS levels are important cellular stressors, closely associated with autophagy induction [38]. To determine how DFO induces autophagy in DPSCs, we detected ROS production in DFO-treated DPSCs. The results showed that the ROS levels were markedly elevated after DFO exposure, and pretreatment with ROS scavenger NAC attenuated DFO-induced autophagy. These results indicated that DFO induces autophagy in DPSCs through ROS generation. Next, we evaluated the effect of ROS in the expression of HIF-1a in DFO-treated DPSCs. Consistent with our previous study [6], DFO could induce accumulation of HIF-1a. Furthermore, inhibition of ROS generation significantly attenuated DFO-induced HIF-1a expression, indicating that DFO could up-regulate the expression of HIF-1a through ROS generation. Importantly, we discovered that NAC also attenuated the expression of BNIP3, which is a hallmark of mitophagy (autophagy targeting mitochondria) and regulated by HIF-1a. Numerous studies have demonstrated that up-regulation of BNIP3 mediated by HIF-1a could activate the autophagic process by releasing Beclin 1 from Bcl2/Beclin 1complex $[18,23]$. Thus, we further investigated the expression of HIF-1a/BNIP3 pathway during DFO treatment. Significant up-regulation of HIF-1a and BNIP3 was observed during DFO treatment. All these evidence suggested that DFO might induce autophagy through activation of HIF-1a/BNIP3 pathway in a ROS-dependent manner in DPSCs.

\section{Conclusion}

The results of the present study showed that autophagy is activated by DFO in DPSCs. Autophagy is responsible for DFO-induced cell migration, differentiation and mineralization in DPSCs, and DFO could induce autophagy in part through HIF-1a/BNIP3 pathway in a ROSdependent manner. Our study suggested that DFO might be a potential therapeutic agent for vital pulp therapy, and an increased understanding of autophagy in DPSCs might facilitate the development of an effective mechanism for dental tissue engineering.

\section{Acknowledgements}

This study was financially supported by the National Natural Science Foundation of China (grant no. 81271134, 81300867), the Specialized Research Fund for the Doctoral Program of Higher Education (grant no. 20130073110013), and Shanghai Summit \& Plateau Disciplines. The authors declare no potential conflicts of interest with respect to the authorship and/or publication of this article. 


\section{Cellular Physiology Cell Physiol Biochem 2017;43:2535-2547 \begin{tabular}{l|l|l} 
and Biochemistry & Dublished online: November 02, 2017 & $\begin{array}{l}\text { (c) } 2017 \text { The Author(s). Published by S. Karger AG, Basel } \\
\text { www.karger.com/cpb }\end{array}$
\end{tabular} \\ Wang et al.: Deferoxamine-Induced Autophagy in the Repair Ability of Dental Pulp Stem Cells}

\section{Disclosure Statement}

None declared.

\section{References}

1 Ratajczak J, Bronckaers A, Dillen Y, Gervois P, Vangansewinkel T, Driesen RB, Wolfs E, Lambrichts I, Hilkens P: The Neurovascular Properties of Dental Stem Cells and Their Importance in Dental Tissue Engineering. Stem Cells Int 2016;2016:9762871.

2 Paino F, La Noce M, Giuliani A, De Rosa A, Mazzoni S, Laino L, Amler E, Papaccio G, Desiderio V, Tirino V: Human DPSCs fabricate vascularized woven bone tissue: a new tool in bone tissue engineering. Clin Sci (Lond) 2017;131:699-713.

3 Chen L, Zheng L, Jiang J, Gui J, Zhang L, Huang Y, Chen X, Ji J, Fan Y: Calcium Hydroxide-induced Proliferation, Migration, Osteogenic Differentiation, and Mineralization via the Mitogen-activated Protein Kinase Pathway in Human Dental Pulp Stem Cells. J Endod 2016;42:1355-1361.

4 Ito K, Matsuoka K, Matsuzaka K, Morinaga K, Inoue T: Hypoxic condition promotes differentiation and mineralization of dental pulp cells in vivo. Int Endod J 2015;48:115-123.

5 Li L, Zhu YQ Jiang L, Peng W, Ritchie HH: Hypoxia promotes mineralization of human dental pulp cells. J Endod 2011;37:799-802.

6 Jiang L, Peng WW, Li LF, Du R, Wu TT, Zhou ZJ, Zhao JJ, Yang Y, Qu DL, Zhu YQ: Effects of deferoxamine on the repair ability of dental pulp cells in vitro. J Endod 2014;40:1100-1104.

7 Shen X, Wan C, Ramaswamy G, Mavalli M, Wang Y, Duvall CL, Deng LF, Guldberg RE, Eberhart A, Clemens TL, Gilbert SR: Prolyl hydroxylase inhibitors increase neoangiogenesis and callus formation following femur fracture in mice. J Orthop Res 2009;27:1298-1305.

8 He C, Klionsky DJ: Regulation mechanisms and signaling pathways of autophagy. Annu Rev Genet 2009;43:67-93.

-9 Mizushima N, Komatsu M: Autophagy: renovation of cells and tissues. Cell 2011;147:728-741.

10 Yang JW, Zhu LX, Yuan GH, Chen YX, Zhang L, Zhang L, Chen Z: Autophagy appears during the development of the mouse lower first molar. Histochem Cell Biol 2013;139:109-118.

11 Lee YH, Lee HY, Kim TG, Lee NH, Yu MK, Yi HK: PPARgamma Maintains Homeostasis through Autophagy Regulation in Dental Pulp. J Dent Res 2015;94:729-737.

12 Zhou Q Liu H, Sun Q Zhang L, Lin H, Yuan G, Zhang L, Chen Z: Adenosine monophosphate-activated protein kinase/mammalian target of rapamycin-dependent autophagy protects human dental pulp cells against hypoxia. J Endod 2013;39:768-773.

13 Li L, Zhu YQ Jiang L, Peng W: Increased autophagic activity in senescent human dental pulp cells. Int Endod J 2012;45:1074-1079.

14 Hocking LJ, Whitehouse C, Helfrich MH: Autophagy: a new player in skeletal maintenance? J Bone Miner Res 2012;27:1439-1447.

15 Pullarkat V, Meng Z, Donohue C, Yamamoto VN, Tomassetti S, Bhatia R, Krishnan A, Forman SJ, Synold TW: Iron chelators induce autophagic cell death in multiple myeloma cells. Leuk Res 2014;38:988-996.

16 Chung JH, Kim YS, Noh K, Lee YM, Chang SW, Kim EC: Deferoxamine promotes osteoblastic differentiation in human periodontal ligament cells via the nuclear factor erythroid 2-related factor-mediated antioxidant signaling pathway. J Periodontal Res 2014;49:563-573.

17 Bohensky J, Shapiro IM, Leshinsky S, Terkhorn SP, Adams CS, Srinivas V: HIF-1 regulation of chondrocyte apoptosis: induction of the autophagic pathway. Autophagy 2007;3:207-214.

18 Schroedl C, McClintock DS, Budinger GR, Chandel NS: Hypoxic but not anoxic stabilization of HIF-1alpha requires mitochondrial reactive oxygen species. Am J Physiol Lung Cell Mol Physiol 2002;283:L922-931.

19 Bronckaers A, Hilkens P, Fanton Y, Struys T, Gervois P, Politis C, Martens W, Lambrichts I: Angiogenic properties of human dental pulp stem cells. PLoS One 2013;8:e71104.

20 Gu S, Ran S, Qin F, Cao D, Wang J, Liu B, Liang J: Human Dental Pulp Stem Cells via the NF-kappaB Pathway. Cell Physiol Biochem 2015;36:1725-1734.

21 Ktistakis NT: Monitoring the Localization of MAP1LC3B by Indirect Immunofluorescence. Cold Spring Harb Protoc 2015;2015:751-755. 
Wang et al.: Deferoxamine-Induced Autophagy in the Repair Ability of Dental Pulp Stem Cells

22 Klionsky DJ, Abdelmohsen K, Abe A, et al.: Guidelines for the use and interpretation of assays for monitoring autophagy (3rd edition). Autophagy 2016;12:1-222.

23 Chinnadurai G, Vijayalingam S, Gibson SB: BNIP3 subfamily BH3-only proteins: mitochondrial stress sensors in normal and pathological functions. Oncogene 2008;27:S114-127.

24 Hentze MW, Muckenthaler MU, Andrews NC: Balancing acts: molecular control of mammalian iron metabolism. Cell 2004;117:285-297.

25 Baschant U, Rauner M, Bulycheva E, Weidner H, Roetto A, Platzbecker U, Hofbauer LC: Wnt5a is a key target for the pro-osteogenic effects of iron chelation on osteoblast progenitors. Haematologica 2016; 101:14991507.

-26 Mancias JD, Wang X, Gygi SP, Harper JW, Kimmelman AC: Quantitative proteomics identifies NCOA4 as the cargo receptor mediating ferritinophagy. Nature 2014;509:105-109.

27 Wu Y, Li X, Xie W, Jankovic J, Le W, Pan T: Neuroprotection of deferoxamine on rotenone-induced injury via accumulation of HIF-1 alpha and induction of autophagy in SH-SY5Y cells. Neurochem Int 2010;57:198205.

28 Moon JH, Jeong JK, Park SY: Deferoxamine inhibits TRAIL-mediated apoptosis via regulation of autophagy in human colon cancer cells. Oncol Rep 2015;33:1171-1176.

29 Kenific CM, Wittmann T: Autophagy in adhesion and migration. J Cell Sci 2016;129:3685-3693.

30 Yamanaka-Tatematsu M, Nakashima A, Fujita N, Shima T, Yoshimori T, Saito S: Autophagy induced by HIF1alpha overexpression supports trophoblast invasion by supplying cellular energy. PLoS One 2013;8:e76605.

-31 Yang JW, Zhang YF, Wan CY, Sun ZY, Nie S, Jian SJ, Zhang L, Song GT, Chen Z: Autophagy in SDF-1alphamediated DPSC migration and pulp regeneration. Biomaterials 2015;44:11-23.

-32 Pierrefite-Carle V, Santucci-Darmanin S, Breuil V, Camuzard O, Carle GF: Autophagy in bone: Self-eating to stay in balance. Ageing Res Rev 2015;24:206-217.

-33 Liu F, Fang F, Yuan H, Yang D, Chen Y, Williams L, Goldstein SA, Krebsbach PH, Guan JL: Suppression of autophagy by FIP200 deletion leads to osteopenia in mice through the inhibition of osteoblast terminal differentiation. J Bone Miner Res 2013;28:2414-2430.

-34 Nollet M, Santucci-Darmanin S, Breuil V, Al-Sahlanee R, Cros C, Topi M, Momier D, Samson M, Pagnotta S, Cailleteau L, Battaglia S, Farlay D, Dacquin R, Barois N, Jurdic P, Boivin G, Heymann D, Lafont F, Lu SS, Dempster DW, Carle GF, Pierrefite-Carle V: Autophagy in osteoblasts is involved in mineralization and bone homeostasis. Autophagy 2014;10:1965-1977.

-35 Wang F, Jiang Y, Huang X, Liu Q Zhang Y, Luo W, Zhang F, Zhou P, Lin J, Zhang H: Pro-Inflammatory Cytokine TNF-alpha Attenuates BMP9-Induced Osteo/ Odontoblastic Differentiation of the Stem Cells of Dental Apical Papilla (SCAPs). Cell Physiol Biochem 2017;41:1725-1735.

-36 Pei F, Wang HS, Chen Z: Autophagy regulates odontoblast differentiation by suppressing NF-kappaB activation in an inflammatory environment. Cell Death Dis 2016;7:e2122.

37 Ozeki N, Hase N, Higuchi N, Hiyama T, Yamaguchi H, Kawai R, Matsumoto T, Nakata K, Mogi M: Gelatin scaffold combined with bone morphogenetic protein-4 induces odontoblast-like cell differentiation involving integrin profile changes, autophagy-related gene 10, and Wnt5 sequentially in human induced pluripotent stem cells. Differentiation 2016;93:1-14.

-38 Poillet-Perez L, Despouy G, Delage-Mourroux R, Boyer-Guittaut M: Interplay between ROS and autophagy in cancer cells, from tumor initiation to cancer therapy. Redox Biol 2015;4:184-192. 